\title{
EURRECA consultation report: what guidance is most needed for developing and updating micronutrient recommendations?
}

\author{
J.P. Lambert ${ }^{1}$ and M. A. Ashwell ${ }^{2}$ \\ ${ }^{1}$ Lambert Nutrition Consultancy, 5 Britwell Road, Watlington OX49 5JS, UK and ${ }^{2}$ Ashwell Associates (Europe), \\ Ashwell Street, Ashwell SG7 5PZ, UK
}

The EURRECA Network of Excellence is working towards the development of aligned micronutrient recommendations or reference values (RV) across Europe. Fundamental to this objective is to standardise methodologies, starting from the process of assessing current RV and setting up panels of experts all the way through to the communication about correct usage of newly-published values. Between these two extremes, common methodologies for the evaluation of the available scientific data are essential. The consultation process aimed to prioritise the types of guidance that are developed in the EURRECA 'toolkit' ${ }^{\text {(1) }}$.

A questionnaire was developed by the authors, following initial expert consultation, and sent electronically during summer 2008 to ninety individuals known to be involved in the development and use of micronutrient RV in Europe and globally. The questionnaire was divided into four sections: the planning process for development or updating RV; the active stage of their development; actions after they have been published; helping others to use them. Respondents were asked to rank each type of guidance from 5 (extremely useful) to 1 (not very useful). Forty-seven (52\%) returned completed questionnaires and average scores were calculated for each question.

The highest scores $(4.0+)$ indicated where guidance was most needed. For planning, these results were guidance on: the best way to assess the 'status quo' of RV (e.g. searchable database of those currently used in Europe and the rest of World and information on how they were derived); process i.e. where to start, what to cover, setting priorities (which nutrients, which population groups) and how to take into consideration the diverse needs of the users; making the whole process transparent. For the active stage, guidance thought to be important was: how to get valid and robust data on micronutrient intakes to use as a base for RV; how to get enough robust data on micronutrient status (and information on good biomarkers) to use as a base for RV; how to account for food-related factors, e.g. bioavailability, which affect requirements and RV; how to produce or incorporate results of systematic reviews and identify unbiased and independent reviews; how to eliminate bias etc.; methods to 'weight' the evidence and how to make decisions if evidence is conflicting; use of formats or concepts to convert scientific requirements into RV (e.g. averages, ranges etc.). For helping users, guidance was required on communicating the RV and accompanying codes of practice in order to raise professional and public awareness, making them easy to use in the intended way and producing more practical tools (e.g. food-based dietary guidelines) to help end users. Guidance on actions after publication such as updating RV was considered less important.

These questionnaire results have been extremely useful for helping EURRECA make decisions on prioritising resources. Network members will now continue to produce such guidance, or 'tools'. Such 'tools' will include scientific papers, but will also be in more practical formats such as consensus criteria, searchable databases, 'gold standard' methods, decision trees or checklists, handbooks or aide-memoirs and web-based tools for calculations. Use of these tools, which have been developed in a transparent and systematic manner, will ultimately help to align micronutrient recommendations.

1. Ashwell M, Lambert JP, Alles MS et al. (2008) Eur J Nutr 47, Suppl. 1, 2-16. 